\title{
Subaru surveys for the high-redshift universe
}

\section{Yoshiaki Taniguchi}

\author{
Department of Physics and Engineering, Graduate School of Science, Ehime University, \\ Bunkyo-cho 2-5, Matsuyama 790-8577, Japan \\ email: tani@sgr.phys.sci.ehime-u.ac.jp
}

\begin{abstract}
We present a brief summary of Subaru observations of high- $z$ galaxies. We discuss our future plan to probe galaxies beyond $z=10$.
\end{abstract}

Keywords. galaxies: evolution, galaxies: formation, galaxies: high-redshift

Among the most pressing issues in modern observational cosmology is the question of finding and studying the first generation of galaxies - their formation epoch, their early evolution, and their contribution to cosmic re-ionization. This provides clues towards early star formation activity in the Universe and mass assembly in galaxies as well as the long standing problem of understanding the physical processes responsible for cosmic reionization of intergalactic medium. Furthermore, this provides an independent test for WMAP results which identifies the reionization epoch to be at $z \simeq 11$. This is the only direct observation currently available constraining the epoch of re-ionization.

The Subaru telescope has been used to search for very high- $z$ galaxies for these several years. The very wide field of view of Subaru-SUPRIME-CAM allows us to identify more than 100 galaxies with $z>5 ; z \simeq 5.7$ (e.g., Shimasaku et al. 2006), $z \simeq 6.6$ (e.g., Taniguchi et al. 2005; Kashikawa et al. 2006), and $z \simeq 7$ (Iye et al. 2006; Hu et al. 2007). In order to extend the search for galaxies at $z>10$, we are promoting to use our custom made near-infrared narrow-band filter (NB2296) on Subaru-MOIRCs to carry out an ultra-deep survey to search for Ly $\alpha$ emitting galaxies at $z \simeq 18$. The central wavelength of this filter is $2296 \mathrm{~nm}$, with a bandwidth corresponding to $23 \mathrm{~nm}$. Therefore, this filter can detect Ly $\alpha$ emission from galaxies at $z \approx 17.9 ; z_{\min }=17.8$ and $z_{\max }=18.0$. The relatively large field of view of Subaru-MOIRCS $\left(4^{\prime} \times 7^{\prime}\right)$ allows a wide-area and ultra-deep survey, rather unprecedented at near-IR wavelengths. Although a re-ionization redshift of $z \simeq 9$ is found by WMAP, it is expected that there are Population III sources out to $z \simeq 30$.

\section{References}

Hu, E. M., Cowie, L. L., \& Kakazu, Y. 2007, these JD07 proceedings, p. 252

Iye, M., Ota, K., Kashikawa, N., et al. 2006, Nature, 443, 186

Kashikawa, N., Shimasaku, K., Malkan, M. A., et al. 2006, ApJ, 648, 7

Shimasaku, K., Kashikawa, N., Doi, M., et al. 2006, PASJ, 58, 313

Taniguchi, Y., Ajiki, M., Nagao, T., et al. 2005, PASJ, 57, 165 